\title{
Available Transfer Capability Evaluation by Decomposition
}

\author{
Mohamed Shaaban, Student Member, IEEE \\ Yixin Ni, Senior Member, EEE \\ Center for Electrical Energy Systems \\ Department of Electrical and Electronic Engineering \\ The University of Hong Kong \\ Hong Kong SAR, CHINA
}

Felix F. Wu, Fellow, IEEE

\begin{abstract}
A central issue of running a successful electric power market is the evaluation of the associated available transfer capability (ATC) representing the room available for trading. Due to the need to post and update ATC values at regular intervals, the underlying calculation method should be moderately fast with acceptable accuracy. This paper proposes the evaluation of ATC by using Benders decomposition. The problem is first broken up into a master problem expressing the steady state operating condition and subproblems for the contingent conditions. Each subproblem is solved independently and a linear constraint using Lagrange multipliers of the subproblem is generated and added to the master problem. The proposed decomposition scheme is applied to IEEE 30 bus system with satisfactory results as compared with the distribution factors method.
\end{abstract}

Keywords: deregulation, power systems, optimal power flow, Benders decomposition, available transfer capability

\section{INTRODUCTION}

Restructuring in the electricity industry is well underway in some parts of the world. These initiatives were compellingly supported by the economic benefits materialized from the restructuring of natural gas, airlines and telecommunications etc. As the wheell rolls on, serious problems are arising. Some are predictable, others not. In general, the instability of electricity combined with very little demand elasticity and the need for real time supply/demand balancing to keep the grid stable have made the restructuring of electricity much more challenging than other industries. Early implementation of restructuring has indicated that, market power among generators represents an imminent and ongoing concern than has been anticipated before. Moreover, the assumption that power imports will flow from low cost generators to high cost areas is not valid for some case such as the transmission congestion and operation limit violation. As a result, the role of transmission capacity is likely to be even more important than previously suggested.

The Federal Energy Regulatory Commission (FERC) rulings mandated the nondiscriminatory open access for the transmission network and the calculation of available transfer capability (ATC) for each control area [1]. ATC measures the transfer capability remaining in the physical system to engage in further transactions above already committed uses [1]. ATC evaluates the usable amount of the transmission network that is accessible to the interconnected system and could be transferred from the starting point to the end point of a path. ATC is mathematically defined as the total transfer capability (TTC) less the transmission reliability margin (TRM), less the sum of existing transmission commitments and capacity benefit margin (CBM). The exposition of the components of ATC is presented in [1]. Since no framework were established to determine these components, TTC is commonly addressed as the basis for ATC evaluation

ATC determination is a nontrivial task as it requires consideration of generation dispatch, system configuration, base scheduled transfers, system contingencies, and projected customer demand $[1,2]$. The ATC values for the next hour and for each hour into the future would be placed on a public domain site known as the open access same-time information system (OASIS) to be operated by an independent system operator (ISO) [1]. Utility engineers must continuously compute and update hourly and daily ATC values [3].

Distribution factors based on linear D.C. power flow methods were proposed to calculate ATC in [3, 4]. Because of the relative ease coupled with the mild computational burden involved in computing these factors, they have found widespread application in the industry [2]. However, in view of their limitations, the future of using such methods in the competitive market environment is doubtful. Continuation power flow (CPF) that incorporate the effects of reactive power flows, voltage limits, and voltage collapse as well as the traditional thermal loading effects was reported in [5]. Since CPF increases the loading factor in discrete steps, it can give conservative transfer capability results. In [6] an optimal power flow approach was proposed taking into account voltage impacts and reactive power. This method could overcome the conservativeness of $\mathrm{CPF}$ and give maximum transfer capability estimates. However, contingency considerations were not addressed.

This paper proposes the evaluation of ATC by using Benders decomposition. Benders decomposition algorithm was developed for solving nonlinear objective/constraints [7]. It decomposes the problem into two levels: the master level and the slave level. The two levels interact with each other until convergence. The master problem here represents the steady state operating condition while the subproblems represent the contingencies. Each subproblem is solved 
independently and a linear constraint using Lagrange multipliers of the subproblem is generated and added to the master problem. The proposed decomposition scheme is applied to IEEE 30 bus system and results were compared favorably with its counterpart from the distribution factors method.

\section{MATHEMATICAL FORMULATION}

Available transfer capability (ATC) measures the transfer capability remaining in the physical system that is available for further trading. ATC determination requires the evaluation of transmission lines thermal limits, voltage deviation at system buses, transient stability and voltage collapse limits. ATC is eventually bounded by the most restrictive limits among them all. The following assumptions are made throughout the study:

(a) The base case power flow of the system is feasible and corresponds to a stable operating point.

(b) The load and generation patterns vary very slowly so that the system transient stability is not jeopardized.

(c) The system has sufficient damping to keep within steady state stability limit.

(d) Bus voltage limits are reached before the system reaches the nose point and loses voltage stability.

The preceding assumptions signify that both transient stability and voltage collapse limits are not concerned. The $\mathrm{N}$ - 1 steady state security criteria will be applied subject to power balance equations, line flow limits and voltage deviation limits.

The Problem is formulated in the form:

$$
\operatorname{Max} . \sum_{s=1}^{\mathrm{ng}} P_{G s}(u)
$$

s.t.

$$
\begin{gathered}
g_{0}(x, u)=0 \\
h_{0}(x, u) \leq 0 \\
g_{k}\left(x_{k}, u_{k}\right)=0 \\
h_{k}\left(x_{k}, u_{k}\right) \leq 0 \\
i=1,2, \ldots, n c
\end{gathered}
$$

where $g_{0}$, and $h_{0}$ are the system equality and inequality constraints at the steady state operating condition. $\mathrm{ng}$ is the number of generators engaged in the specified transaction that ATC is required to be calculated for. $P_{G s}$ is the generation at bus $s . u$ is the control variable. $x$ is the state variables comprising voltage magnitudes and angles. The subscript $k$ for the contingency case $k . g_{k}$, and $h_{k}$ are the equality and inequality constraints for the contingency case $k$. $n c$ is the number of contingencies. The constraints for contingency cases are similar to the normal operation case with the exception of one element of the system being out. This objective function aims to calculate ATC by augmenting the total generation of the source or supply area where the load of the receiving or sink is inherently enlarged. In this paper, we take the incremental change of the total load at the receiving area, from its base case magnitude, as ATC between these two areas when all the loads are served by that particular supply area.

Detailed expressions of the objective function and the constraints for the security constrained ATC evaluation are given below:

$$
\operatorname{Max} . \sum_{s=1}^{\mathrm{ng}} P_{G s}(u)
$$

s.t.

$$
\begin{gathered}
P_{i}^{k}-V_{i}^{k} \sum_{j=1}^{N} V_{j}^{k}\left(G_{i j} \cos \theta_{i j}+B_{i j} \sin \theta_{i j}\right)=0 \\
Q_{i}^{k}-V_{i}^{k} \sum_{j=1}^{N} V_{j}^{k}\left(G_{i j} \sin \theta_{i j}-B_{i j} \cos \theta_{i j}\right)=0 \\
k=0 \text { (normal case), } \\
k=1,2, \ldots, n c \text { (contingencies) } \\
P_{G s}^{\min } \leq P_{G s}^{k} \leq P_{G s}^{\max }, s \in S \\
Q_{G m}^{\min } \leq Q_{G m}^{k} \leq Q_{G m}^{\max }, m=1,2, . ., M \\
P_{L d}^{k} \leq P_{L d}^{\max }, d \in D \\
V_{i}^{\min } \leq V_{i}^{k} \leq V_{i}^{\max }, i=1,2, . ., N \\
0 \leq I_{i j}^{k} \leq I_{i j}^{\max }, i, j=1,2, . ., N, i \neq j
\end{gathered}
$$

The variables are defined as follows:

$P_{G s}:$ is the generation at bus $s$

$P_{i}$, and $Q_{i}$ : are the active and reactive power injection at bus $i$. $N$ is the total number of network buses.

$V_{i} \angle \theta_{i}:$ is the voltage at bus $i$

$\theta_{i j}=\theta_{i}-\theta_{j}$ : voltage angle between bus $i$ and $j$

$G_{i j}+j B_{i j}$ is the corresponding conductance and susceptance in system Y-matrix.

$P_{G s}^{\max }$ and $P_{G s}^{\min }:$ are the upper and lower limits of the generator active power at bus $s . S$ represents the sending area where the generators can increase their output. Other generators in the system remain virtually constant. The number of generators in $S$ is $n g$.

$Q_{G m}^{\max }$ and $Q_{G m}^{\min }$ : are reactive power limits for generator $m . M$ is the number of generators in the network.

$P_{L d}$ : is the load at bus $d . D$ designates the sink area where the transaction is taking place. Load increase is performed at constant power factor. The number of loads at the sink is $N d$. 
$P_{L d}^{\max }:$ is the upper limit of the load because of the distribution facility capacity.

$I_{i j}$ and $I_{t j}^{\max }$ : are the actual and maximum current of line $i-j$ respectively.

The number of constraints, either equalities or inequalities, in (2) is proportional to the number of contingency cases analyzed. The nonlinearity of (2) coupled with the large number of constraints makes the use of non-decomposition algorithms susceptible to potential computational difficulties. This incites the use of a decomposition-based scheme.

\section{BENDERS DECOMPOSITION SCHEME}

The basic idea of Benders decomposition is to break down the problem into a master problem and a set of subproblems, which are linked through coupled variables [7-9]. The subproblem solves each contingency case independently with the coupled variables temporarily fixed. The subproblem solution provides the Lagrange multipliers associated with the coupling variables to the master problem. The master problem, derived via nonlinear duality theory, in turn determines whether the coupled variables need modification to be used subsequently in the subproblems again. As the iterations proceed, the two problems react reciprocally until certain convergence tolerance is achieved. To solve the nonlinear optimization problem (1) using Benders decomposition, coupling variables should be first established between the base case steady state operating point and the contingency cases as given below

$$
\begin{array}{cc} 
& \text { Max. } \sum_{s=1}^{\mathrm{ng}} P_{G s}(u) \\
\text { s.t. } & g_{0}(x, u)=0 \\
h_{0}(x, u) \leq 0 \\
g_{k}\left(x_{k}, u_{k}\right)=0 \\
h_{k}\left(x_{k}, u_{k}\right) \leq 0 \\
\left|u_{0}-u_{k}\right| \leq \operatorname{Ramp}\left(P_{G s}\right) \\
k=I, 2, \ldots, n c
\end{array}
$$

Where $\mathrm{u}_{0}$ is the base case generator output considering no contingencies. Ramp $\left(P_{G s}\right)$ is the ramping constraints of each generator in the supply area. The ramping constraints enable the system to perform corrective rescheduling in case of emergency control. If the ramping equals to zero, this corresponds to the preventive rescheduling mode.

\section{A. Subproblems}

The objective of the subproblems is to minimize the deviation of the post contingency control from the base case operating point such that the coupling constraints are satisfied. If the objective function equals to zero or certain tolerance, then a feasible operating point has been reached. Each subproblem supplies the master problem with the violation amount and the Lagrange multiplier associated with its coupling constraints. The subproblem is formulated as:

$$
\operatorname{Min} \sum_{s=1}^{n g} \alpha_{s}
$$

s.t.

$$
\begin{gathered}
g_{k}\left(x_{k}, u_{k}\right)=0 \\
h_{k}\left(x_{k}, u_{k}\right) \leq 0 \\
\left|u^{m}-u_{k}\right|-\alpha_{s} \leq \operatorname{Ramp}\left(P_{G s}\right) \\
\alpha_{s} \geq 0 \\
k=1,2, \ldots, n c \\
s=1,2, \ldots, n g
\end{gathered}
$$

$\alpha_{\mathrm{s}}$ is a scalar that measures the coupling constraint violation associated with the post-contingency control $u_{k}$. $\mathbf{u}^{\mathrm{m}}$ corresponds the $\mathrm{m}$-th iteration control, i.e., generator active power outputs. In the first iteration, $u^{\mathrm{m}}$ will be the base case generator outputs. It will be updated in latter iterations reflecting the new operating point.

\section{B. Master Problem}

The Lagrange multiplier vector $\lambda$ and the coupling constraint violations $\alpha_{s}^{*}$ for the particular contingency $k$ are fed back to the master problem. The two are used to form the linear Benders cut as shown below. This cut represents the marginal change of the post-fault contingency case as a linear function of the base case operating point $(x, u)$.

$$
\begin{gathered}
\text { Min }-\left\{\sum_{s=1}^{n g} P_{G s}(u)\right\} \\
g(x, u)=0 \\
h(x, u) \leq 0 \\
\alpha_{s}^{*}+\lambda\left(u-u^{m}\right) \leq 0
\end{gathered}
$$$$
\text { s.t. }
$$

The solution of problem (5) is equivalent to the solution of problem (3). As each new candidate solution is generated, new Benders cuts are appended to the master problem. Thus the master problem increases in size as the algorithm proceeds.

\section{Algorithm}

Because of the large number of constraints involved in solving the master problem (5), a relaxation strategy is intuitively the most suitable. Practical power systems include large number of contingencies, However, most of these contingencies do not violate the system operating conditions. Therefore it would be constructive to filter out harmless contingencies as outlined in the algorithm below:

a) Initialize $n=1$ (counter) 
Relax all security-related constraints as well as the set of Benders cuts.

b) Solve the relaxed master optimization problem.

$$
\operatorname{Max} . \sum_{s=1}^{\mathrm{ng}} P_{G s}(u)
$$

s.t.

$$
\begin{aligned}
& g_{0}(x, u)=0 \\
& h_{0}(x, u) \leq 0
\end{aligned}
$$

c) The optimal solution $u^{m}$ gives the lower bound of the optimal solution, since problem (6) is a relaxed version of the global solution. Determine ATC for the relaxed problem.

d) For the operating conditions determined in step $c$, use any fast screening method to remove non-violating contingencies from the subproblems.

e) Given $u^{\mathrm{m}}$ solve the optimal subproblems (4). Check whether the optimal solution of (4) for all the subproblems is within the specified tolerance. If so, ATC calculated in step $c$, is the global solution and terminate the computation.

f) If the solution of (4) is greater than the tolerance, then $\mathrm{u}^{\mathrm{k}}$ gives the upper bound of the solution.

g) To improve the lower bound estimate of the function, Benders cuts are generated and the new constraints are added to problem (6), which will take the form given in (5).

h) Advance iteration counter $n+l$ and go to step $b$ to solve the master problem and obtain new $\mathbf{u}^{\mathrm{m}}$.

\section{TEST RESULTS}

IEEE 30 bus system is used to study the proposed decomposition scheme to calculate the available transfer capability (ATC). The system has 6 generators, 41 lines, and 3 areas, where each area has 2 generators as shown in Fig. 1. Initially, each area satisfies the native load from its own generation resources with area 1 baring the system losses of $2.5 \mathrm{MW}$. The transfer from area 1 to area 2 is reported here. Area 1 has generators \#1 and \#2. The base case ATC, corresponding to (6), is $104.2 \mathrm{MW}$. The binding constraints were the thermal limits of lines 25-27, and 10-17. Both generators were at their maximum output level in this case. Receiving area's maximum demand above the base case loading is taken as ATC.

To examine the efficacy of the proposed decomposition scheme, a single contingency case is considered using a nondecomposition-based method, as in (3), and the decomposition algorithm. Table 1 . demonstrates the results for the outage of line 6-9. For comparison purposes, the preventive control mode was assumed throughout this study.

Table 1. Outage of line $6-9$

\begin{tabular}{|l|c|}
\hline Method & ATC, MW \\
\hline Benders decomposition & 77.1 \\
\hline Non-decomposition & 76.6 \\
\hline
\end{tabular}

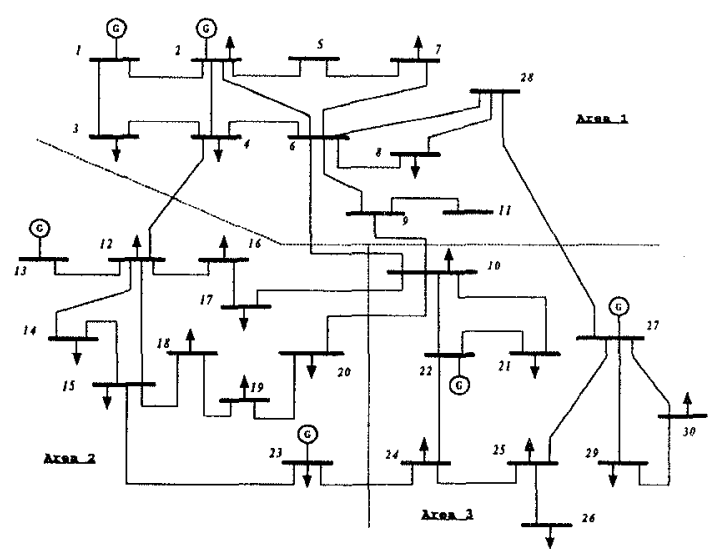

Fig. 1. IEEE 30 bus test system

Results in table 1. show that, Benders decomposition method yields accurate results.

Alternatively, ATC is calculated using dc linear sensitivity analysis. In this case, reactive power/voltage considerations are neglected and transmission line thermal limits are the only effective restraints. Active power transfer distribution factors (PTDFs), which relate the transaction amount to the line power flow, are computed. The base case ATC using distribution factors was $78.9 \mathrm{MW}$ with line 25-27 is the binding constraint. Moreover, line outage distribution factors (LODFs) are calculated as well to measure the redistribution of power over the network when an outage occurs. From these factors, linear estimates of ATC can be readily obtained [3]. Table 2 portrays the results for evaluating ATC between area 1 and area 2 using distribution factors for some cases as compared to their counterpart using Benders decomposition.

Table 2. ATC values using distribution factors and Benders decomposition

\begin{tabular}{|c|c|c|c|}
\hline \multirow{2}{*}{ Case } & \multirow{2}{*}{ Line outage } & Distrib. factors & Benders decomp. \\
\cline { 3 - 4 } & $1-3$ & 70.9 & 91.4 \\
\hline 1 & $2-4$ & 70.7 & 88.9 \\
\hline 2 & $4-12$ & 40.5 & 52.8 \\
\hline 3 & &
\end{tabular}

The results given in table 2 indicate that the error between the distribution factors based ATC evaluation and Benders decomposition, more accurate, method ranges between $20 \%$ to $40 \%$. These results show how linear sensitivity analysis can diminish trading across the system significantly underestimating ATC. This is explicitly due to the disregard 
of reactive power and bus voltage optimization. Full ac analysis is therefore indispensable for the evaluation of ATC.

\section{CONCLUSIONS}

Available transfer capability (ATC) evaluation under steady state security conditions using Benders decomposition was investigated in this paper. The problem was formulated as an optimization problem with all system normal operating conditions and contingency conditions represented in detail. The problem is decomposed into two levels: the master level and the slave level. The two levels interact with each other until convergence. The master problem represents the steady state operating condition while the subproblems represent the contingencies. Each subproblem is solved independently and a linear constraint using Lagrange multipliers of the subproblem is generated and added to the master problem. The proposed decomposition scheme is applied to the IEEE 30 bus system and the computer results were compared favorably with its counterpart from the distribution factors method.

\section{ACKNOWLEDGEMENT}

The research is supported by the National Key Basic Research Special Fund (No. G1998020305), P. R. China, to whom sincere acknowledgement is expressed.

\section{REFERENCES}

[1] Transmission Transfer Capability Task Force, "Available Transfer Capability Definitions and Determination", North American Electric Reliability Council, Princeton, NJ, June 1996.

[2] B.S. Gisin, M.V. Obessis, and J.V. Mitsche, "Practical methods for transfer limit analysis in the power industry deregulated environment", Proceedings the 1999 Power Industry Computer Applications Conference, pp. 261267.

[3] R.D. Christie, B.F. Wollenberg, and I. Wangensteen, "Transmission management in the deregulated environment', IEEE Proceedings, vol. 88, no. 2, February 2000 , pp. 170-195.

[4] G.C. Ejebe, J.G. Waight, M. Santos-Nieto and W.F. Tinney, "Fast calculation of linear available transfer capability", Proceedings the 1999 Power Industry Computer Applications Conference, pp. 255-260.

[5] G.C. Ejebe, J. Tong, J.G. Waight, J.G. Frame, X. Wang, and W.F. Tinney, "Available transfer capability calculations", IEEE Trans. Power Systems, Vol. 13, no. 4, November 1998, pp. 1521-1527.

[6] M. Shaaban, Y.X. Ni, and F.F. Wu, "TTC calculation using SQP method considering reactive power and voltage impacts", submitted to IEEE Trans. Power Systems.
[7] A.M. Geoffrion, "Generalized Benders decomposition", Journal of Optimization theory and Applications, Vol. 10, no. 4,1972 , pp. $237-260$.

[8] A. Monticelli, M.V.F. Pereira, and S. Granville, "Security-constrained optimal power flow with postcontingency corrective rescheduling", IEEE Trans. Power Systems, Vol. PWRS-2, no. 1, February 1987, pp. 175 182.

[9] Y.Y. Hong, and M.T. Weng, "Optimal short-term real power scheduling in a deregulated competitive market", Electric Power Systems Research, Vol. 54, 2000, pp. 181188.

\section{BIOGRAPHIES}

Mohamed Shaaban (S'99) received both his B.Sc. and M.Sc. in Electrical Engineering from Suez Canal University, Egypt in 1990 and 1996 respectively. He is currently pursuing his Ph.D. in the University of Hong Kong. His research interests are power system analysis, stability, and power markets.

Yixin $\mathrm{Ni}$ (SM'94) received her B. Eng., M. Eng., and Dr. Eng. from Electrical Engineering Department, Tsinghua University, P.R. China in 1968, 1981, and 1983 respectively. Her research interests are in power system analysis, stability, control, power electronics applications, and power markets. She was a Professor at Tsinghua University and is now with the University of Hong Kong.

Felix F. Wu (M'73, SM'86, F'89) joined the University of Hong Kong as the Chair Professor of Electrical Engineering in September 1995. Prior to that, he was Professor and vice Chairman, Department of Electrical Engineering and Computer sciences, University of California, Berkeley, the same institute where he received his $\mathrm{Ph} . \mathrm{D}$. degree. His research interests are power system planning and operation, including economics and reliability in system planning, realtime security assessment, and design of energy management systems and distribution automation. Recently he has been involved in the design of industry restructuring and electricity pricing. 